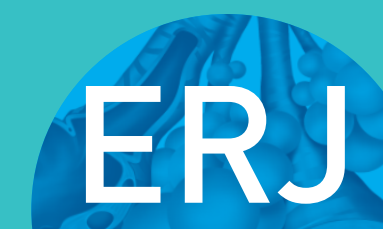

open research
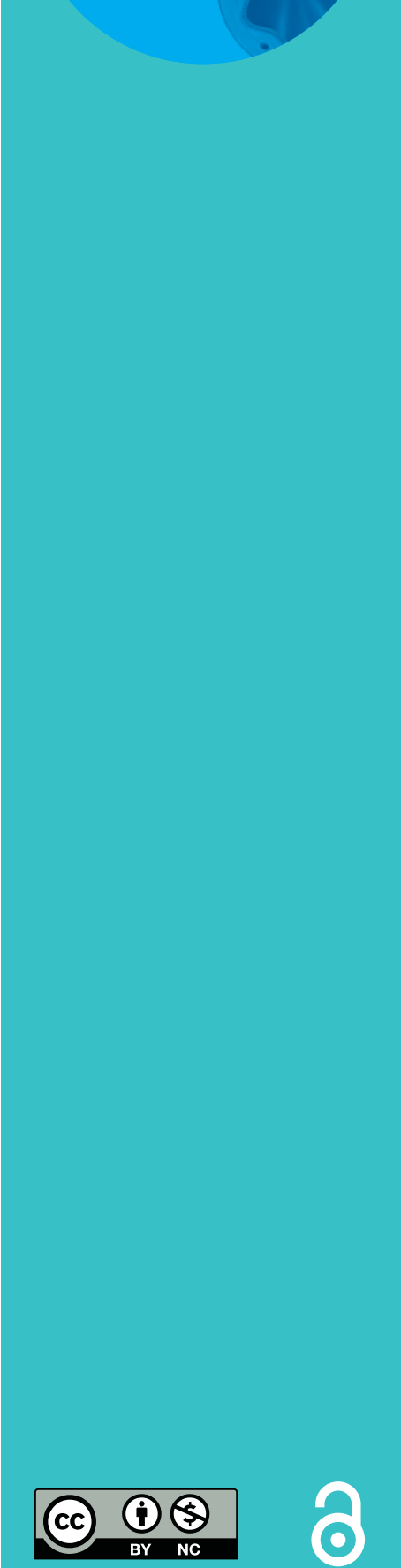

\section{Tuberculosis control, and the where and why of artificial intelligence}

\author{
Riddhi Doshi (1) ${ }^{1}$, Dennis Falzon², Bruce V. Thomas ${ }^{3}$, Zelalem Temesgen ${ }^{4}$, \\ Lal Sadasivan ${ }^{5}$, Giovanni Battista Migliori $\mathbb{1}^{6}$ and Mario Raviglione ${ }^{2}$
}

Affiliations: ${ }^{1}$ Center for Public Health and Health Policy, University of Connecticut Health Center, Farmington, CT, USA. ${ }^{2}$ Global TB Programme, World Health Organization, Geneva, Switzerland. ${ }^{3}$ The Arcady Group, Richmond, VA, USA. "Mayo Clinic, Rochester, MN, USA. ${ }^{5} \mathrm{PATH}$, Washington, DC, USA. ${ }^{6}$ WHO Collaborating Centre for TB and Lung Diseases, Maugeri Care and Research Institute, Tradate, Italy.

Correspondence: Riddhi Doshi, 263 Farmington Ave, MC 6030, Farmington, CT 06030-6030, USA.

E-mail: rpduconnagmail.com

ABSTRACT Countries aiming to reduce their tuberculosis (TB) burden by 2035 to the levels envisaged by the World Health Organization End TB Strategy need to innovate, with approaches such as digital health (electronic and mobile health) in support of patient care, surveillance, programme management, training and communication. Alongside the large-scale roll-out required for such interventions to make a significant impact, products must stay abreast of advancing technology over time. The integration of artificial intelligence into new software promises to make processes more effective and efficient, endowing them with a potential hitherto unimaginable. Users can benefit from artificial intelligence-enabled pattern recognition software for tasks ranging from reading radiographs to adverse event monitoring, sifting through vast datasets to personalise a patient's care plan or to customise training materials. Many experts forecast the imminent transformation of the delivery of healthcare services. We discuss how artificial intelligence and machine learning could revolutionise the management of TB.

@ERSpublications

Tuberculosis control, and the where and why of artificial intelligence http://ow.ly/GwuY30bCXkJ

Cite this article as: Doshi R, Falzon D, Thomas BV, et al. Tuberculosis control, and the where and why of artificial intelligence. ERJ Open Res 2017; 3: 00056-2017 [https://doi.org/10.1183/ 23120541.00056-2017].

Received: April 242017 | Accepted: April 302017

Conflict of interest: None declared.

The content of this work is $\odot$ the authors or their employers. Design and branding are $\odot$ ERS 2017. This article is open access and distributed under the terms of the Creative Commons Attribution Non-Commercial Licence 4.0. 


\section{Introduction}

The World Health Organization (WHO) estimates that there were 10.4 million new cases of tuberculosis (TB) and 1.8 million deaths caused by TB in 2015, a poignant reminder of the threat that this epidemic continues to pose to global health despite concerted efforts to curb it in recent decades [1]. The WHO's End TB strategy maps out the global response required between 2016 and 2035 in order to reduce worldwide TB incidence by $90 \%$ and TB mortality by $95 \%$ [2]. Digital technologies can make global efforts to end TB more effective and efficient [3]. Access to the internet and affordable mobile devices is becoming increasingly widespread in low- and middle-income countries. In the past 2 years, WHO and its partners identified a set of priority electronic and mobile health products and concepts which were advantageously positioned to have a high impact on different domains of TB care and prevention [4]. Target product profiles (TPPs), consisting of descriptions of how these tools should work, were developed in order to communicate the product attributes to all partners involved in advancing them. The TPPs are intended to be dynamic documents which are updated regularly to incorporate the latest in the technology and remain relevant to TB practitioners and patients who should benefit from the finished product.

Digital technologies can support efforts against TB in four ways: patient care, surveillance, programme management and e-learning [3]. A number of currently available digital products already address one or more of these functions, but to achieve the ambitious goals of the End TB strategy these tools need to be rolled out more extensively. Moreover, their performance needs to be augmented by incorporating innovations such as artificial intelligence (the branch of computer science concerned with the automation of intelligent behaviour [5]) and machine learning (the subfield of computer science that gives computers the ability to learn without being explicitly programmed [6]) into the future successors of today's technology [7]. Artificial intelligence is already embedded in common applications that recognise voice and visual patterns, such as speech transcription software and computer-aided diagnostic platforms.

In this article, we present some leading digital technologies being applied in TB control and discuss the value that artificial intelligence could add to the next generation of technology.

\section{Patient care}

Adherence to medication is one of the most important challenges facing TB patients. The need to travel regularly to health facilities over many months for treatment observation influences compliance to treatment for chronic conditions such as TB [8]. Long-term adherence can be motivated through patient-provider communication, which can be improved using informatics. Several promising digital technologies are now being introduced for this purpose. Electronic medication monitors capable of transmitting short message service (texts) have been successfully deployed in low-resource settings with a high TB burden [9]. In addition, virtual directly observed treatment via video (VOT or VDOT), which involves the provider observing a TB patient taking medication via video sent over a smartphone or tablet computer has been shown to be feasible under socially challenging conditions, as access to affordable devices and to broadband internet extends to most places where TB patients live [10-12]. Both of these technologies still require substantial input from health workers to interpret the signals they send. Moreover, the risk of breach of privacy, such as that associated with the transmission of video over internet, has been a concern. These risks could be reduced through encryption. However, smarter methods are being employed. The potential of artificial intelligence to recognise patterns and gestures reliably and consistently could add huge value to the toolset currently available to the practitioners [13]. For instance, in VDOT, software could be enabled to identify the unique features of a particular person taking a specific medication; when combined with the timestamp of the event and the mobile phone number, a distinct signature can be generated and transmitted as a small data packet to the caregiver. Beyond TB, this technique could be of value in other situations requiring close clinical monitoring.

While treatment adherence has attracted much focus, digital technology could improve TB patient care through other approaches. For instance, "clip-on" hardware can convert a smartphone into a clinical instrument $[14,15]$. Cognitive computing (systems that learn at scale, reason with purpose and interact with humans naturally [16]) could power algorithms within clinical decision support systems to help clinicians with diagnostic and treatment decisions [17]. They could help, for instance, in the causality assessment of adverse events through the recognition of patterns and the association of various data. Artificial intelligence could enable rapid access to previously unreachable masses of data. These could include diverse sources ranging from cloud-based datasets that are not usually roped into clinical decision-making, as well as rich knowledge bases which usually remain locked up inside the individual patient's genome [18]. Access to this information helps both the human operator and the machine to learn. If an advanced artificial intelligence application can be incorporated into a mobile-based clinical decision support application, TB workers worldwide would not only benefit from its use, but also enrich it by the data and feedback they supply. Such a personalised approach to care is bound to challenge 
time-honoured practices, such as how new treatment regimens have been traditionally introduced in clinical care on the basis of observations extrapolated from drug trial participants. The demand for these resource-intensive studies, which can take several years to complete, could thus decrease.

\section{Surveillance}

Attempts to record and track TB patient information electronically have a long history, and recent years have seen a global expansion and diversification of software being deployed for this purpose, often in open-source format [19-21]. Another critical milestone has been internet penetration. Technology acceptance over time has been impressive. For instance, in the early 1990s, TB officers in southern India started to email their TB surveillance reports to the higher administrative level. In later years, public health facilities experimented with different electronic medical records systems. More recently, and in support of the notification of TB by public and private practitioners, which became mandatory in 2012, a nationwide, online, disaggregated TB reporting platform (NIKSHAY) was implemented with the immediate result of facilitating the increasing reporting of cases $[22,23]$. In China, the TB information management system provides another example of the large-scale use of digital technology for TB surveillance [20]. The system has evolved over time and offers a number of benefits, such as the flagging of "at-risk" TB patients for the evaluation of drug resistance and the direct reporting to the national infectious disease register to ensure timeliness and accuracy and to limit duplication of work.

Artificial intelligence could add value to the review of disaggregated patient records. Where unique personal identifiers are commonly unavailable an intelligent system could still trace an individual person's "signature" over time within large datasets. This would be greatly important in TB surveillance, for instance to trace people with clinical manifestations suggestive of TB or disease relapse or adverse drug reactions and to complete the monitoring of mortality and the linkage with HIV. In addition, this approach could identify predictors of treatment failure, which in turn could be useful in patient care as well as TB drug development [24, 25]. Artificial intelligence has been proposed as a means to detect outbreaks of TB early on [26].

\section{Programme management}

Information has always been central to planning and management. In TB programmes the handling of diagnostic data is crucial to maximise coverage of services, identify inequities and malfunctions and limit waste. "Connected diagnostics" is becoming a flagship concept in TB programme management, helping to monitor diagnostic machines remotely and to consolidate the results data. Successful implementation of such systems in low-resource settings has been given a boost by the advent of molecular diagnostics that generate their results digitally [27]. The next generation of products could go a step further by helping to interpret results data and match them with those from other diagnostic processes. Artificial neural networks have been applied to clinical situations that still pose a problem for practitioners, such as microscopy sputum smear-negative $\mathrm{TB}$ and pleural $\mathrm{TB}$, in settings where diagnostic options are limited $[28,29]$. The potential contribution of computerisation to radiology, another important field in TB diagnostics was first recognised decades ago [30]. Nonetheless, the automated detection of TB on digital chest radiographs has yet to become more clinically relevant [31].

\section{e-Learning}

e-Learning uses electronic media and devices for training and communication [32]. In its different forms, ranging from digital texts that replace paper to advanced online interaction, e-learning is rewriting the textbook approach to human resource development and patient education. "Gamification" and augmented reality environments are among the novel techniques being tested to simulate problem-solving environments for the learner [33]. Artificial intelligence is being used to emulate the human tutor's ability both to customise the method of knowledge acquisition, as well as to prioritise content based on the learner's capacity, preference and knowledge gaps. Developing e-learning materials requires a multidisciplinary mix of skills to ensure that the content is not only pertinent, correct and up to date, but also attractive in format and appropriately sized for the media being used to deliver it. It thus incurs a substantial cost. Artificial intelligence can automate the laborious classification of texts and images needed to create the content of an app, thus speeding up the process and lowering costs. Moreover, artificial intelligence could empower the automated grading of student assessments, increasing the value of online courses and releasing time for tutors to devote to other tasks.

\section{The (near) future?}

The huge capacity of digital tools to compute and store data has profound implications in the healthcare sector, where massive amounts of data are generated and the prospect of sifting them efficiently to improve patient outcomes is particularly appealing. Many of the healthcare processes streamlined by 
digital technologies still require supervision by the human expert, albeit to varying degrees. Classical examples are online discussion groups dedicated to clinical problems such as drug-resistant $\mathrm{TB}$, which are moderated by human expertise [34-36]. While robots have grown to outperform their teachers in certain tasks, they have not yet supplanted medical specialists. This is the next frontier, which is set to be breached as machines progress from supportive tools to take a bigger share of the driving seat in medical decision-making. It appears that the role of the healthcare worker is destined to become primarily that of the "gatekeeper" of clinically relevant data. Medical specialties that are commonly solicited in pulmonology and TB, such as imaging, microscopy and pathology depend heavily upon the interpretation of disease morphology and may thus be among the ones most "disrupted" by automated pattern recognition. In addition, artificial intelligence can contribute to basic research.

While artificial intelligence has been incorporated in medical innovations since the 1970s [37], forthcoming advances such as cognitive computational systems will mark a radical departure from the previous order. Telemedicine and remote consultations can deliver healthcare via a home computer or mobile device, challenging the conventions of patient-carer relationships. Portable and wearable devices equipped with artificial intelligence-enhanced software can monitor patients and issue alerts fatigue-free to a degree which would be impossible even where trained workers are available. These developments will strengthen the trend towards home-based care and reduce demand on "brick and mortar" healthcare facilities. The arrival of the smartphone in the past decade has given us a taste of how technology can rapidly transform lay persons into informed clients of healthcare services. This trend will keep expanding in future and will equip patients and their caregivers with more options to interact and to make the best possible clinical decisions.

\section{Acknowledgements}

All authors contributed to the ideation and writing of this manuscript, and agree with the conclusions drawn. D. Falzon and M. Raviglione are staff members of the World Health Organization (WHO). They alone are responsible for the views expressed in this publication and they do not necessarily represent the decisions or policies of WHO. The designations used and the presentation of the material in this publication do not imply the expression of any opinion whatsoever on the part of WHO concerning the legal status of any country, territory, city or area, or of its authorities, nor concerning the delimitation of its frontiers or boundaries.

\section{References}

1 World Health Organization (WHO). Global Tuberculosis Report 2016 (WHO/HTM/TB/2016.13). Geneva, WHO, 2016. www.who.int/tb/publications/global_report/en/ Date last accessed: May 26, 2017. Date last updated: 2016.

Uplekar M, Weil D, Lönnroth K, et al. WHO's new end TB strategy. Lancet 2015; 385: 1799-1801.

3 World Health Organization (WHO)/European Respiratory Society. Digital Health for the End TB Strategy: an Agenda for Action (WHO/HTM/TB/2015.21). Geneva, WHO, 2015. www.who.int/tb/areas-of-work/digital-health/ Digital_health_EndTBstrategy.pdf Date last accessed: May 26, 2017. Date last updated: 2015.

4 Falzon D, Timimi H, Kurosinski P, et al. Digital health for the End TB Strategy: developing priority products and making them work. Eur Respir J 2016; 48: 29-45.

5 Luger GF, Stubblefield WA. Artificial Intelligence: Structures and Strategies for Complex Problem Solving. 2nd edn. Redwood City, Benjamin/Cummings Publishing Company, 1993; p. 740.

6 Samuel AL. Some studies in machine learning using the game of checkers. 1959. IBM Journal of Research and Development. http://dl.acm.org/citation.cfm?id=1661924\&CFID=940986350\&CFTOKEN=25154997 Date last accessed: May 26, 2017.

7 Marr B. How Machine Learning, Big Data and AI are Changing Healthcare Forever. Forbes. 2016 www.forbes. $\mathrm{com} /$ sites/bernardmarr/2016/09/23/how-machine-learning-big-data-and-ai-are-changing-healthcare-forever/print/ Date last accessed: January 16, 2017. Date last updated: 2016

8 Munro SA, Lewin SA, Smith HJ, et al. Patient adherence to tuberculosis treatment: a systematic review of qualitative research. PLoS Med 2007; 4: e238.

9 Liu $\mathrm{X}$, Lewis JJ, Zhang $\mathrm{H}$, et al. Effectiveness of electronic reminders to improve medication adherence in tuberculosis patients: a cluster-randomised trial. PLoS Med 2015; 12: e1001876.

10 Garfein RS, Collins K, Muñoz F, et al. Feasibility of tuberculosis treatment monitoring by video directly observed therapy: a binational pilot study. Int J Tuberc Lung Dis 2015; 19: 1057-1064.

11 Story A, Garfein RS, Hayward A, et al. Monitoring therapy compliance of tuberculosis patients by using video-enabled electronic devices. Emerg Infect Dis 2016; 22: 538-540.

12 Sinkou H, Hurevich H, Rusovich V, et al. Video observed treatment for tuberculosis patients in Belarus: findings from the first programmatic experience. Eur Respir J 2017; 49: 1602049.

13 AiCure. Advanced Medication Adherence Solutions. https://aicure.com/wp-content/uploads/2016/12/Shafner_et_ al_Vancouver_Feb_2017.pdf Date last accessed: May 26, 2017. Date last updated: 2017.

14 Peer S, Fagan JJ. Hearing loss in the developing world: evaluating the iPhone mobile device as a screening tool. S Afr Med J 2014; 105: 35-39.

15 Breslauer DN, Maamari RN, Switz NA, et al. Mobile phone based clinical microscopy for global health applications. PLoS One 2009; 4: e6320.

16 Kelly JE. Computing, Cognition and the Future of Knowing. How Humans and Machines are Forging a New Age of Understanding. New York, IBM Global Services. www.research.ibm.com/software/IBMResearch/multimedia/ Computing_Cognition_WhitePaper.pdf Date last accessed: May 26, 2017. Date last updated: October 2015.

17 Kalyanpur A, Murdock J. Unsupervised Entity-Relation Analysis in IBM Watson. Proceedings of the Third Annual Conference on Advances in Cognitive Systems. 2015: Article 12. 
18 Malin JL. Envisioning Watson as a rapid-learning system for oncology. J Oncol Pract 2013; 9: 155-157.

19 Nadol P, Stinson KW, Coggin W, et al. Electronic tuberculosis surveillance systems: a tool for managing today's TB programs. Int J Tuberc Lung Dis 2008; 12: Suppl. 1, 8-16.

20 Huang F, Cheng S, Du X, et al. Electronic recording and reporting system for tuberculosis in China: experience and opportunities. J Am Med Inform Assoc 2013; 21: 938-941.

21 Collect, Manage, Visualize and Explore your Data | DHIS 2. www.dhis2.org/ Date last accessed: May 26, 2017. Date last updated: 2017

22 Revised National Tuberculosis Control Programme. About Nikshay. http://nikshay.gov.in/AboutNikshay.htm Date last accessed: May 26, 2017. Date last updated: 2017.

23 Roy S, Rai DR, Suresh G. Tuberculosis - a notifiable disease. J Indian Med Assoc 2012; 110: 728-731.

24 Swaminathan S, Pasipanodya JG, Ramachandran G, et al. Drug concentration thresholds predictive of therapy failure and death in children with tuberculosis: bread crumb trails in random forests. Clin Infect Dis 2016; 63: Suppl. 3, S63-S74.

25 Modongo C, Pasipanodya JG, Magazi BT, et al. Artificial intelligence and amikacin exposures predictive of outcomes in multidrug-resistant tuberculosis patients. Antimicrob Agents Chemother 2016; 60: 5928-5932.

26 El-Solh AA, Hsiao C-B, Goodnough S, et al. Predicting active pulmonary tuberculosis using an artificial neural network. Chest 1999; 116: 968-973.

27 Global Laboratory Initiative. GLI Quick Guide to TB Diagnostics Connectivity Solutions. www.stoptb.org/wg/gli/ assets/documents/gli_connectivity_guide.pdf Date last accessed: May 26, 2017. Date last updated: 2016.

28 de O Souza Filho JB, de Seixas JM, Galliez R, et al. A screening system for smear-negative pulmonary tuberculosis using artificial neural networks. Int J Infect Dis 2016; 49: 33-39.

29 Seixas JM, Faria J, Souza Filho JB, et al. Artificial neural network models to support the diagnosis of pleural tuberculosis in adult patients. Int J Tuberc Lung Dis 2013; 17: 682-686.

30 Lusted LB. Logical analysis in roentgen diagnosis. Radiology 1960; 74: 178-193.

31 Pande T, Cohen C, Pai M, et al. Computer-aided detection of pulmonary tuberculosis on digital chest radiographs: a systematic review. Int J Tuberc Lung Dis 2016; 20: 1226-1230.

32 Sangrà A, Vlachopoulos D, Cabrera N. Building an inclusive definition of e-learning: an approach to the conceptual framework. Int Rev Res Open Distrib Learn 2012; 13: 145-159.

33 Urh M, Vukovic G, Jereb E, et al. The model for introduction of gamification into e-learning in higher education. Procedia Soc Behav Sci 2015; 197: 388-397.

34 Global Health Delivery Online: Improving Health Care Delivery Through Global Collaboration. www.ghdonline. org/ Date last accessed: January 30, 2017. Date last updated: May 25, 2017

35 Blasi F, Dara M, van der Werf MJ, et al. Supporting TB clinicians managing difficult cases: the ERS/WHO Consilium. Eur Respir J 2013; 41: 491-494.

36 D'Ambrosio L, Tadolini M, Centis R, et al. A new free-cost e-service supporting clinicians to manage their difficult-to-treat TB cases: the ERS-WHO TB consilium. J Thorac Dis 2015; 7: 1080-1085.

37 Patel VL, Shortliffe EH, Stefanelli M, et al. The coming of age of artificial intelligence in medicine. Artif Intell Med 2009; 46: 5-17. 